\section{Editorial comment: is essential tremor a neurodegenerative disease?}

\author{
Amos D. Korczyn
}

Published online: 8 August 2012

(C) Springer-Verlag 2012

The question of whether essential tremor (ET) is a neurodegenerative disease or not has been a focus of recent interest, and the two opposing views are very well expressed by the two opponents, Ubaldo Bonuccelli and Monica Kurtis, who presented their cases during a recent CONy Congress and have now written their arguments here.

Both authors base their conclusions on overlapping studies. If so, why is there still a disagreement?

The first issue raised is the spectrum of ET, which includes more than kinetic tremor in many cases. However, this is not a major issue. Amyotrophic lateral sclerosis has been considered a neurodegenerative disease for decades, assuming that it affects only the motor neurons. The recent identification of additional features, such as genetic heterogeneity and autonomic dysfunction (Chandran and Pal 2012) has not changed this substantially.

There is no question that, to some extent, the symptoms in ET are progressive. Nobody is born with ET, and although the first manifestations may appear in early adulthood or later in life, we do not have good long-term prospective follow-up studies that describe the natural history. However, there is little doubt that tremor increases in severity in many patients, rarely to the extent of causing significant incapacity, while in most patients, tremor remains relatively stable, being merely a nuisance of cosmetic rather than clinical significance.

A major point is our incomplete understanding of the pathogenesis of the tremor, and it is still unclear whether it

A. D. Korczyn $(\bowtie)$

Tel Aviv University Medical School, Ramat Aviv,

Tel Aviv, Israel

e-mail: amoskor@tau.ac.il

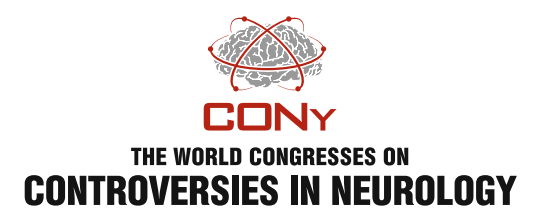

originates in dysfunc-

tion of the cerebellum, the superior olives, or in disrupted circuitry. Associated with the non-specific changes which have been described in the limited autopsy material available, it is still impossible to identify and define the underlying processes.

The most important missing data needed to answer the question of whether ET is a neurodegenerative disease are the lack of reliable, long-term, prospective studies, which will include quantitative evaluation of tremor amplitude as well as additional cerebellar, extrapyramidal and nonmotor manifestations. The available epidemiological data are mostly cross sectional, with a significant selection bias.

It is likely that such future studies will result in finding ET to be a heterogeneous disease. There is a lot of data supporting this view: the age of onset (the previous distinction of "senile tremor" from an early onset entity has been discarded, but without scientific evidence that indeed early and late-onset disease constitutes a single nosologic entity); response to drug and alcohol; electrophysiological data (Golan et al. 2004); and finally, genetics and pathology. Of course, once different entities are identified which are now included under the umbrella term of ET, each may have its own etiology and natural history.

\section{References}

Chandran V, Pal K (2012) Essential tremor: beyond the motor features. Parkinson Dis Relat Disord 18:407-414

Golan D, Giladi N, Thorne R, Simon ES (2004) Subdivision of essential tremor patients' according to physiologic characteristics. Acta Neurol Scand 109:292-297 